\title{
Linx
}

Revue des linguistes de l'université Paris X Nanterre

$13 \mid 2020$

Collocations et traditions discursives

\section{Intégrer des méthodologies pour étudier le discours de la presse sur les réfugiés}

Joy Weigand

\section{(2) OpenEdition}

\section{Journals}

Édition électronique

URL : http://journals.openedition.org/linx/3977

DOI : 10.4000/linx.3977

ISSN : 2118-9692

Éditeur

Presses universitaires de Paris Nanterre

Référence électronique

Joy Weigand, «Intégrer des méthodologies pour étudier le discours de la presse sur les réfugiés », Linx [En ligne], 13 | 2020, mis en ligne le 30 mai 2020, consulté le 01 juin 2020. URL : http:// journals.openedition.org/linx/3977 ; DOI : https://doi.org/10.4000/linx.3977

Ce document a été généré automatiquement le 1 juin 2020.

Département de Sciences du langage, Université Paris Ouest 


\title{
Intégrer des méthodologies pour étudier le discours de la presse sur les réfugiés
}

\author{
Joy Weigand
}

\section{Introduction}

1 L'immigration vers l'Europe commence à augmenter à un rythme alarmant en 2007. En 2015, elle atteint le statut de crise lorsque 1,2 million de premières demandes d'asile, soit plus du double de l'année précédente est comptabilisé. Selon le communiqué de presse d'Eurostat (Bourgeais 2016), des 1,2 million de demandes d'asile, $35 \%$ étaient pour l'Allemagne, $14 \%$ la Hongrie, $12 \%$ la Suède, 7 \% l'Autriche, $6 \%$ la France, 3,5 \% le Royaume-Uni, 1,5\% l'Espagne. Elles concernent $58 \%$ d'hommes, $17 \%$ de femmes, et $25 \%$ d'enfants. Depuis 2015, le nombre de demandes d'asile diminue progressivement. Bien que le nombre de nouveaux demandeurs d'asile ait diminué de près de la moitié en 2017, selon le Financial Times (Coco 2018), il reste anormalement élevé, comme le montre la figure suivante. En effet, les données fournies par Eurostat font état de 1,2 million de demandeurs d'asile en 2015, 1,2 million en 2016 et 650000 en 2017. 


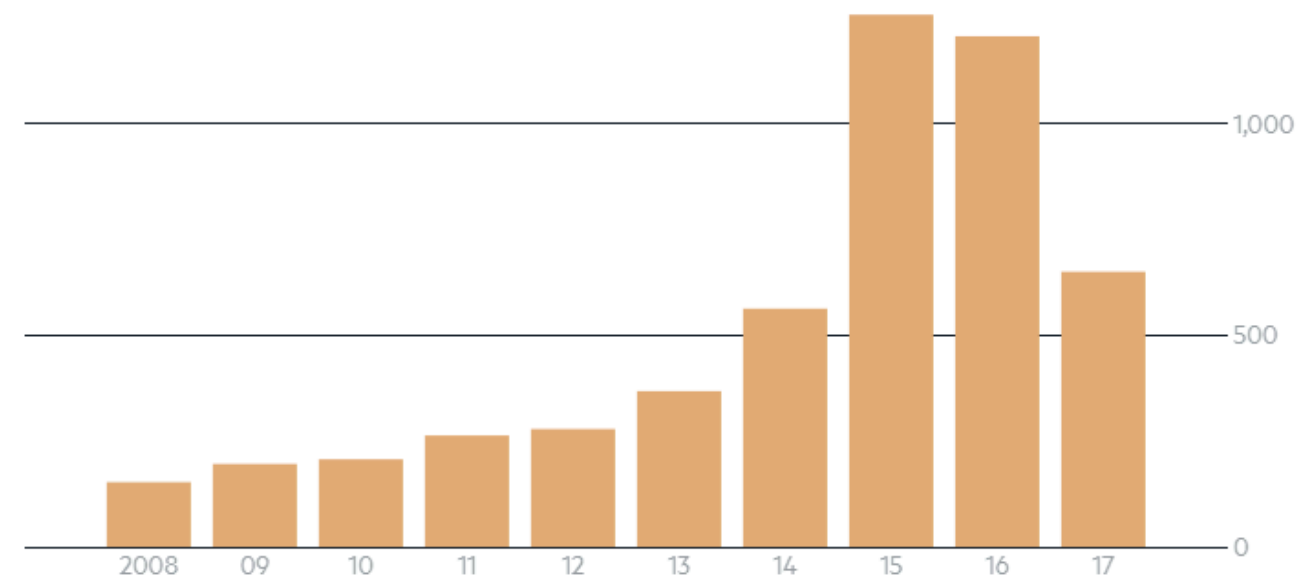

Source: Eurostat

(c) FT

Figure 1. Demandeurs d'asile en 2017 (Euorstat 2018).

2 En 2017, l'Allemagne reçoit $31 \%$ de l'ensemble des premières demandes dans l'UE, soit plus que tout autre pays de l'UE, suivie par l'Italie (20\%), la France (14\%) et la Grèce (9\%) selon Eurostat (2018).

Germany is the most generous EU country to refugees

Total asylum applicants and number of those granted refugee status, per million inhabitants of each EU country (2016)

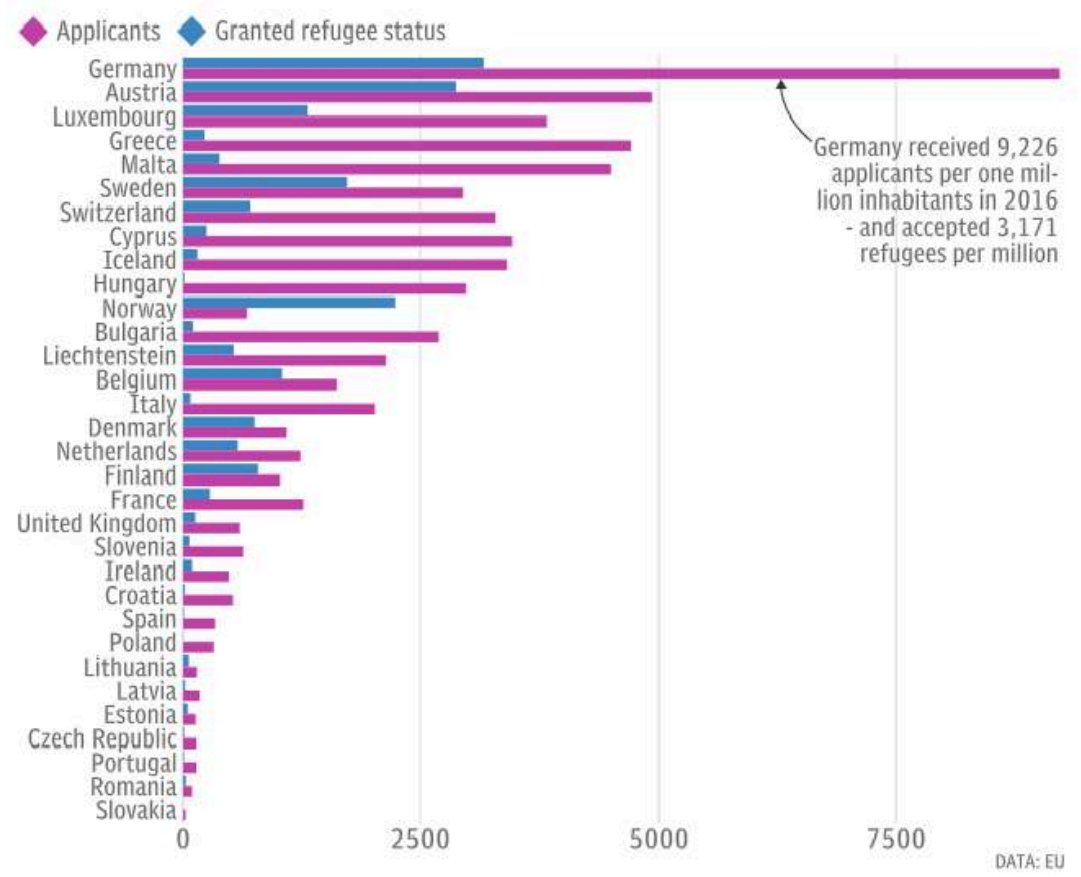

Figure 2. Demandeurs d'asile accordé (Ashley 2016)

3 Il est frappant de constater non seulement le nombre de demandeurs d'asile qui se voient accorder le statut de réfugié, mais aussi de ceux qui se le voient refuser. La 
figure 2 souligne la gravité de cette situation. De nombreux aspects de cette situation sont rapportés dans la presse, qui aborde la question sous différents angles. Il s'agit sans aucun doute d'un phénomène saisissant, qui soulève un certain nombre de questions auxquelles doivent répondre tant sur le plan politique que social la société, les chercheurs et tout citoyen. Cependant, cette recherche se concentrera uniquement sur les questions linguistiques et en particulier sur la méthodologie utilisée pour étudier ce phénomène. Lorsqu'une demande d'asile est acceptée, le demandeur est accepté par le pays d'accueil et nommé légalement réfugié. Cela signifie que la procédure de demande d'asile d'un demandeur d'asile est achevée, qu'elle a abouti et que la personne a été acceptée par le pays d'accueil, puis légalement appelée réfugié, représenté en bleu sur la figure 2. C'est avec ce mot de réfugié que commence mon étude.

4 Selon les résultats d'un sondage réalisé par le Pew Research Center (voir figure 3), de nombreux citoyens des pays d'accueil considèrent les réfugiés comme des menaces. Mais comment cette idée d'un réfugié constituant une menace peut-elle émerger? La presse a-t-elle joué un rôle dans la formation de l'opinion? L'objectif de la recherche est de déterminer le rôle de la presse, à travers les choix linguistiques qu'elle effectue, dans la formation de l'opinion des citoyens des pays d'accueil sur la question des réfugiés, en quatre langues : anglais, allemand, espagnol et français. Cette recherche se concentre sur les collocations et la prosodie sémantique que l'on trouve dans les articles écrits par des auteurs qui peuvent avoir une idéologie particulière à l'esprit. Afin d'étudier le phénomène d'une manière impartiale, l'élément majeur dans la conception de cette recherche est de choisir ou d'élaborer la méthodologie appropriée.

\section{Many Europeans see refugees from Syria and Iraq as a major threat}

$\%$ saying large mumber of refugees leaving Iraq and Syria is a major threat to their country
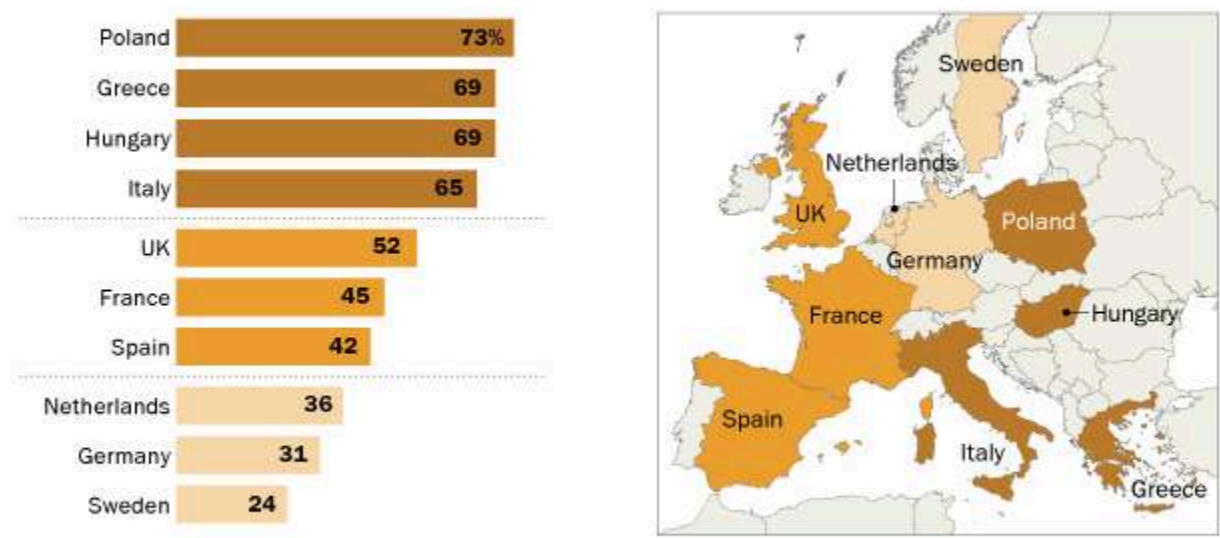

Source: Spring 2016 Global Attitudes Survey PEW RESEARCH CENTER

Figure 3. Opinions européennes sur les réfugiés (Pousther 2016) 


\section{Littérature}

5 Afin de fournir un contexte à cette étude globale, il convient de fournir un aperçu de la recherche sur la collocation et la prosodie sémantique, en s'intéressant notamment à la méthodologie utilisée le cas échéant.

Il existe de nombreuses études linguistiques sur la migration, mais étant pour la plupart monolingues, elles explorent principalement la facette sociolinguistique du discours politique. Quelques-unes seulement portent sur le mot réfugié. Si les études mono-linguistiques et cross-linguistiques portant sur la prosodie sémantique existent, peu d'entre elles se sont penchées, dans une perspective cross-linguistique, sur la collocation, la prosodie sémantique et les thèmes développés par la presse dans les discours concernant les réfugiés.

\section{1 Études mono-linguistiques}

$7 \quad$ L'étude de Baker et McEnery (2005: 222) sur les journaux et les textes britanniques est importante à deux titres. Elle fait la constatation linguistique de l'existence d'un discours négatif sur la prosodie et de la préférence du mot réfugié par rapport à celui de demandeur d'asile. D'un point de vue méthodologique, elle démontre que l'analyse du discours peut être fondée sur le corpus (Baker et McEnery 2005 : 222). Dans le cadre du projet global, Gabrielatos et Baker (2008:5) identifient un certain nombre de catégories d'expressions qui peuvent être évaluées comme négatives, ils soulignent l'existence et le développement de mots absurdes, par exemple, réfugiés illégaux, et la confusion des médias lorsqu'il s'agit de définir des mots comme réfugiés, demandeurs d'asile, immigrants et migrants (Gabrielatos/Baker 2008). Selon Blinder et Allen,

les représentations de la presse correspondent à la perception qu'a le public des migrants, les immigrants illégaux et les demandeurs d'asile déboutés étant les plus souvent décrits dans les journaux à grand tirage et les tabloïds (Blinder/Allen 2016 :

3 , traduit par nous).

Dans son analyse critique du discours sur les demandeurs d'asile immigrants et migrants (RASIM) pendant le conflit des Balkans en 1999 et les élections générales britanniques en 2005, Khosravinik découvre qu'«il existe des similitudes dans les catégories micro linguistiques utilisées dans les représentations de ces groupes et [que] les journaux contribuent tous à une construction similaire de ces personnes, perpétuant des stéréotypes et reproduisant des attitudes négatives» (Khosravinik 2009 : 477, traduit par nous). L'étude d'Al Fajri (2017:389) révèle une représentation commune ainsi que des "discours hégémoniques» centrés uniquement sur les immigrants et leurs collocations, transmettant une représentation négative du mot. En effet, les collocations les plus fréquentes sont négatives, comme l'adjectif collocatif illégal. L'étude d'Ibrahim (2017: 29), portant sur les reportages consacrés à la représentation des migrants et des réfugiés dans les journaux britanniques à la suite des attentats de Cologne, dévoile un discours raciste discriminatoire comprenant des généralisations, par exemple une culture de la violence et autres comme une façon de définir et décrire des réfugiés et migrants. De son côté, Parker cherche à montrer la manière dont les demandeurs d'asile et les réfugiés sont qualifiés dans la presse écrite, tant au Royaume-Uni qu'en Australie; le répertoire prédominant utilisé dans les articles est celui d'envahisseurs indésirables, de déviant ou criminels actifs, "qui sont 
arrivés pour causer des problèmes à la majorité passive » (Parker 2015 : 15, traduit par nous). De même, Moore, étudiant le shopping d'asile, constate dans son analyse critique de la presse britannique ${ }^{1}$ l'utilisation « d'un référent établi dans le lexique du reportage d'asile, potentiellement disponible pour être utilisé à des fins idéologiques lorsque nécessaire" (Moore 2013: 362, traduit par nous). Non pas que le magasinage soit négatif en soi. La contradiction réside dans la juxtaposition presque oxymoronique du mot shopping, qui implique la liberté de choix, avec celui d'asile, associé à la nécessité de chercher protection. Il est intéressant de noter que cette combinaison de mots « n'apparaitt pas dans un article de journal en soi » (Moore $2013: 362$, traduit par nous), mais apparaît, généralement masquée, dans le discours relatif aux demandeurs d'asile :

Ses conditions d'émergence ont été remises en question de façon circonstancielle, tandis que les techniques journalistiques couramment déployées pour souligner sa nouveauté ont même facilité son articulation «de bon sens» dans le discours hégémonique sur l'asile. (Moore 2013 : 362, traduit par nous).

Moore (2013: 137) constate que les médias en ligne utilisent des métaphores plus chargées d'émotion au sujet des réfugiés syriens dans la langue du pays hôte que dans celle des pays non-hôtes.

En 2000, Böke et al. ont examiné la presse de langue allemande en créant un dictionnaire historique du discours à partir d'un corpus sur l'immigration (réfugiés et demandeurs d'asile). Il apparait, dans les articles des cinq journaux allemands étudiés, que «les réfugiés étaient négativement connotés jusqu'au milieu des années 1960 » (Böke et al. $2000: 28$, traduit par nous). Selon Schnörch,

il faut accorder une attention particulière à la description lexicographique dans les dictionnaires allemands, en particulier dans le cas de mots tels que étrangers, réfugiés migrants, demandeurs d'asile, etc. qui sont utilisés dans une controverse avec la langue allemande. (Schnörch $2014: 348$, traduit par nous)

11 Mustafa-Awad et Kirner-Ludwig se penchent spécifiquement sur la représentation des femmes arabes dans la presse en Allemagne et concluent que

leurs stéréotypes récurrents dans les médias sont en train d'être brisés et de nouvelles images les présentent comme des agents du changement et combattants plutôt que victimes passives de leurs sociétés (Mustafa-Awad/Kirner-Ludwig 2017 : 533, traduit par nous).

12 Examinant la presse francophone, Jacquez identifie «trois registres principaux: l'ouverture des frontières/mobilité et les arguments contre l'expulsion, et le droit de rester sur la base de l'intégration sociale » (Jacquez $2015: 1$ ). Son étude des quotidiens français révèle une typologie linguistique des principaux registres utilisés dans la dénonciation des expulsions des immigrants clandestins.

Selon Salas, les expressions décrivant des migrants dans la presse hispanophone peuvent être "regroupées soit dans la catégorie descriptive, soit dans celle des migrants ajoutant un aspect connotatif à la plupart des expressions impliquant des mouvements, et associant les migrants à une crise économique » (Salas 2016: 116, traduit par nous). C'est la première étude qui relie les migrants à la crise économique. Fernández et Corral constatent que :

bien que l'intérêt humain soit généralement mis en avant, les sujets négatifs prévalent dans les journaux espagnols ACB, El País, La Vanguardia et Heraldo de Aragón impliquant le Maghreb. (Fernández et Corral 2016 : 96, traduit par nous).

14 De plus, ils remarquent une disparité entre les journaux : 
Les journaux régionaux se sont engagés sur les questions nationales et régionales avec le travail, la culture et la coexistence. $A C B$ était extrêmement préoccupé par les tensions et les conflits. El País avec des thèmes interculturels, Heraldo de Aragón et $L a$ Vanguardia encadrent leurs textes du point de vue de l'intérêt humain. (Fernández et Corral, 2016:96, traduit par nous)

Hoyer quant à lui relève des informations intéressantes sur la manière dont les médias espagnols évoquent les réfugiés :

L'utilisation des mots «refugiados» (réfugiés), «demandantes de asilo » (demandeurs d'asile) et «migrantes » (migrants) pour désigner les réfugiés syriens, [...] crée une division entre les réfugiés et la société hôte; l'utilisation de mots comme "personas » (personnes) et l'utilisation des noms permet également une identification entre lecteurs et réfugiés. (Hoyer, $2016: 2$, traduit par nous)

Il met ainsi l'accent sur l'absence de réfugiés dans la presse par des entretiens directs.

\section{2 Études cross-linguistiques}

17 Les études cross-linguistiques tendent à se concentrer davantage sur la prosodie sémantique des langues en général et ne se limitent pas toujours aux articles de presse. Elles montrent avec pertinence que les prosodies peuvent varier dans différentes langues. Dans une étude descriptive des prosodies sémantiques du portugais brésilien et de l'anglais, Sardinha (2000: 106) trouve "diverses prosodies» dans ces langues respectives. Xiao et McEnery font les observations suivantes sur le chinois et l'anglais :

La prosodie sémantique et la préférence sémantique peuvent être affectées par des variations morphologiques en anglais mais non en chinois qui ne possède pas les mêmes variations ; [...] de presque synonymes peuvent avoir différentes prosodies, et les modèles de collocation ainsi que les prosodies sémantiques peuvent varier entre catégories de textes. (Xiao et McEnery $2006: 126$, traduit par nous)

L'étude de Tognini-Bonelli sur les fonctions pragmatiques dans un corpus comparable d'italien et d'anglais révèle «de nombreuses différences de comportement de collocation, de préférence sémantique entre ces langues» (Tognini-Bonelli 2001: 138, traduit par nous). Revenant aux médias, Fotopoulos et Kaimaklioti se concentrent sur la presse grecque, allemande et britannique et constatent :

les médias ont une approche uniforme pour couvrir la crise en présentant les réfugiés comme des victimes, avec une grande attention portée à la question tragique des enfants réfugiés (Fotopoulos et Kaimaklioti 2016:273, traduit par nous).

19 Berry et ses collaborateurs se concentrent quant à eux sur la couverture médiatique des réfugiés dans cinq pays européens, l'Espagne, l'Italie, l'Allemagne, le Royaume-Uni et la Suède. Les médias exploitent des thèmes divers et utilisent des mots différents pour décrire les réfugiés :

L'Allemagne et la Suède, par exemple, utilisent massivement les termes "réfugié " ou «demandeur d'asile ", tandis que l'Italie et la presse britannique préfèrent le mot « migrant » et les thèmes humanitaires [sont] plus courants dans la couverture italienne que dans la presse britannique, allemande ou espagnole. (Berry et al. $2016: 255$, traduit par nous)

La presse italienne se concentre quant à elle sur les patrouilles frontalières. Bien que « la voix des gens ordinaires soit bien représentée, contrairement à ce qui se passe en Suède ou en Allemagne, les perspectives des citoyens sur les migrants sont largement négatives et, dans de nombreux cas, xénophobes » (Berry et al. 2016:257, traduit par 
nous). Dans la presse allemande, il existe un clivage gauche-droite visible dans le choix des thèmes, la droite se concentrant sur la politique et le nombre de réfugiés et la gauche sur les tragédies en Méditerranée (Berry et al. 2016: 260). En Suède, les médias évoquent peu la Méditerranée contrairement à la stratégie politique, même s'ils développent un grand nombre de thèmes humanitaires (Berry et al. $2016: 262$ ). C'est au Royaume-Uni que les reportages sont les plus polarisés, la gauche adoptant des thèmes et termes suscitant l'empathie et la droite utilisant des étiquettes plus désobligeantes, en employant des termes comme illégal, immigrant illégal ou migrant illégal (Berry et al. $2016: 253)$.

21 Après avoir donné un aperçu d'une sélection d'études monolingues et crosslinguistiques, comme le reconnaissent Xiao et McEnery, il est «urgent que les chercheurs poursuivent l'étude cross-linguistique de la collocation et de la prosodie sémantique » (Xiao et McEnery, 2006 : 139, traduit par nous). Mon étude est une étude cross-linguistique qui vise à compléter la recherche existante et à élargir les informations disponibles sur la collocation et la prosodie sémantique.

\section{Méthodologie}

Il est important de déterminer la meilleure approche pour analyser le discours des médias sur les réfugiés. Mon approche consiste à examiner les collocations du mot réfugié ainsi que la prosodie sémantique. Firth propose de définir la collocation ainsi :

Collocations of a given word are statements of the habitual or customary places of that word (Firth $1968: 181$ ).

Ce n'est pas un concept nouveau. Le mot est utilisé pour la première fois en 1957 par Firth (1957). Les deux principales caractéristiques de la collocation sont la cooccurrence de modèles de mots (Firth 1968; Sinclair et al. 1970; Kilgarriff 2005; McEnery et al., 2006) et l'aspect quantitatif et statistique (Krishnamurthy 2000; Halliday 1966; Greenbaum 1974 ; Sinclair 1991; Hoey 1991; Stubbs 1995 ; Partington 1998 ; McEnery et Wilson 2001 ; Hunston 2002). Cette définition minimaliste n'est pas sans poser problème. Elle recouvre des composés nominaux, des verbes à particules et des expressions idiomatiques. Toutefois, elle fournit une base générale pour saisir l'utilisation du mot réfugié dans son contexte.

Selon Louw (2000: 56), la prosodie sémantique fait référence à une forme de signification qui s'établit par la proximité d'une série cohérente de collocations, souvent caractérisées comme positives ou négatives, et dont la fonction première est l'expression de l'attitude de son locuteur ou de son auteur face à une situation pratique. On la qualifie également de discours, de prosodie pragmatique ou d'associations sémantiques. La prosodie sémantique est importante car de telles associations, prises en charge par des réseaux neuronaux pour traiter l'information, peuvent être manipulées par la presse, intentionnellement ou non, et présenter une version altérée des faits.

Avant d'examiner de plus près la collocation et la prosodie sémantique, il était important d'examiner les méthodes et les approches appropriées à l'étude. L'analyse du discours et la linguistique de corpus ont toutes deux prévalu dans leur application aux études sur les migrations et les réfugiés au cours des dernières années. Il est de plus en plus courant de combiner l'analyse critique du discours et la linguistique du corpus afin de bénéficier d'une analyse à plusieurs niveaux. De plus, l'analyse du discours critique 
assistée par corpus, dérivée de cette tendance croissante à combiner des éléments de l'analyse critique du discours et de linguistique de corpus, prend de l'importance. C'est cette méthodologie que j'ai choisi d'utiliser dans cette étude.

\subsection{L'analyse critique du discours}

Selon Fairclough (2003: 125), l'analyse critique du discours comprend deux formes d'analyses. La première est l'analyse interdiscursive des genres, des discours et des styles et de la façon dont ils s'articulent, dont ils sont texturés ensemble. La seconde est l'analyse des formes textuelles que prennent ces mélanges de genres, de discours et de styles. Elle implique une analyse linguistique et d'autres formes d'analyse sémiotique, comme celle des images et du langage visuel. L'analyse critique du discours n'est pas pertinente pour étudier une unité linguistique en soi, mais pour étudier des phénomènes sociaux qui sont nécessairement complexes et qui exigent donc une approche multidisciplinaire et multiméthodique (Wodak $2009: 2$, traduit par nous). Les concepts clés de l'analyse critique du discours sont l'idéologie, le pouvoir, l'hégémonie et l'identité. L'analyse critique du discours permet l'utilisation de méthodes qualitatives pour l'examen des données fournies par les corpus.

Une analyse critique s'intéresserait non seulement aux éléments et aux processus linguistiques qui existent dans un texte ou un ensemble de textes, mais aussi à expliquer pourquoi et dans quelles circonstances et avec quelles conséquences les producteurs du texte ont fait des choix linguistiques spécifiques parmi plusieurs autres options qu'une langue donnée peut offrir. (Baker et al. $2008: 281$, traduit par nous)

Il convient d'utiliser cette approche pour examiner le discours tenu sur les réfugiés, car les thèmes fondamentaux sur lesquels s'appuie l'analyse critique du discours idéologie, pouvoir, identité, hégémonie - sont récurrents dans les corpus. En outre, l'examen de ces thèmes est crucial pour comprendre la relation de la presse avec les réfugiés ainsi que les choix linguistiques réalisés par celle-ci dans les articles qu'elle produit.

28 Les discours sont des façons de représenter des aspects du monde - les processus, les relations et les structures du monde matériel, le «monde mental des pensées, des sentiments, des croyances, etc. dans les mondes sociaux» (Fairclough 2003: 124, traduit par nous). Selon van Dijk (1993, cité dans Flowerdew 2013 : 182, traduit par nous): "il est possible d'analyser les structures linguistiques et les stratégies discursives d'un discours afin de mettre au jour les luttes de pouvoir, les inégalités sociales et autres formes de problèmes sociaux et politiques en cause ». L'analyse critique du discours nous permet d'examiner la relation complexe du langage et du pouvoir :

L'analyse critique du discours rend compte de la façon dont les structures discursives - qui sont établies à travers divers schémas et structures linguistiques fonctionnent de manière spécifique pour transmettre les cognitions sociales (comment les gens pensent) - ce qui contribue à son tour au développement de structures sociales d'inégalité et d'injustice du pouvoir dans la société. (Flowerdew $2013: 183$, traduit par nous) 


\subsubsection{L'idéologie}

L'idéologie est curieuse car elle représente des idées et pourrait être vaste, mais dans un autre sens elle pourrait avoir un sens très spécifique. Fairclough souligne un point intéressant, en écrivant qu'"il n'est pas rare de trouver des mots comme idéologie décrits comme 'insignifiants' parce qu'ils ont tant de sens» (Fairclough 2003: 77, traduit par nous). L'idéologie est importante dans les relations de pouvoir. Elle est avant tout une représentation des aspects du monde dont on peut démontrer qu'ils contribuent à établir et à maintenir des relations de pouvoir, de domination et d'exploitation (Fairclough 2003 : 123). Les textes utilisent souvent des techniques et des dispositifs linguistiques pour véhiculer des idéologies économiques, politiques et sociales. Cette étude tente de révéler à travers les collocations utilisées les indices idéologiques exprimés par divers membres de la presse.

\subsubsection{Le Pouvoir}

L'une des principales différences entre le discours médiatique et la communication en face à face, soulignée dans Language and Power de Fairclough (Fairclough $2003: 49$ ) est le caractère unilatéral de l'interaction des participants. L'un écrit, les autres lisent. De plus, celui qui exerce le pouvoir demeure invisible : écrivain, éditeur, gouvernement, organisation, le véritable auteur du discours n'est pas toujours décelable. Selon Wodak, " pour l'analyse critique du discours, le langage n'est pas puissant en soi - il acquiert du pouvoir par l'utilisation que les gens puissants en font » (Wodak 2009: 10, traduit par nous).

31 L'influence qu'exerce la presse, selon van Dijk (1996, cité dans Gabrielatos / Baker $2008: 8$, traduit par nous), peut être considérée comme une forme de "pouvoir social », c'est-à-dire comme "le contrôle exercé par un groupe ou une organisation (ou ses membres) sur les actions et/ou les esprits d'un autre groupe, limitant ainsi la liberté des actions des autres, ou influençant leurs connaissances, attitudes ou idéologies ».

On parle souvent des effets causés par l'individu et la société, une relation de cause à effet qui ne peut pas toujours être tracée directement avec régularité. Mais les pouvoirs causaux qui façonnent les textes sont aussi bien des pouvoirs d'agence que de structure (Fairclough 2003: 126).

Plutôt que de définir le pouvoir, van Dijk présente les aspects politiques, socioculturels et économiques plus généraux et présupposés de la domination (Clegg 1989; Lukes 1974, 1986 ; Wrong 1979) :

1. Le pouvoir est une propriété des relations entre les groupes sociaux, les institutions ou les organisations. Par conséquent, seul le pouvoir social, et non le pouvoir individuel, est considéré ici.

2. Le pouvoir social se définit par le contrôle exercé par une organisation de groupe (ou ses membres) sur les actions et/ou les esprits d'un autre groupe, ce qui limite la liberté d'action des autres, ou influence leurs connaissances, attitudes ou idéologies.

3. Le pouvoir d'un groupe ou d'une institution spécifique peut être « distribué », et peut être limité à un domaine ou à une portée sociale spécifique, comme celui de la politique, des médias, de l'ordre public, de l'éducation ou des affaires des entreprises, ce qui donne lieu à différents « centres » de pouvoir et à des élites qui contrôlent ces centres. 
4. La domination est ici comprise comme une forme d'abus de pouvoir social, c'està-dire comme l'exercice illégitime, sur le plan juridique ou moral, d'un contrôle sur autrui dans son propre intérêt, ce qui entraîne souvent des inégalités sociales.

5. Le pouvoir repose sur l'accès privilégié à des ressources sociales précieuses, telles que la richesse, l'emploi, le statut ou, en fait, à un accès préférentiel au discours public et à la communication.

6. Le pouvoir social et la domination sont souvent organisés et institutionnalisés de manière à permettre un contrôle plus efficace et à permettre des formes routinières de reproduction du pouvoir.

7. La domination est rarement absolue; elle est souvent graduelle et peut se traduire par plus ou moins de résistance ou de contre-pouvoir de la part des groupes dominés. (T. van Dijk 2013 : 93-113, traduit par nous)

Le pouvoir dont il est ici question est la «manipulation de modèles mentaux d'évènements sociaux par l'utilisation de structures discursives spécifiques, telles que structures thématiques, titres, style, figures rhétoriques, stratégies sémantiques, etc. $»^{2}$ qui peuvent «aboutir, faute de données alternatives, à la création de modèles préférentiels de situations spécifiques, qui peuvent à leur tour être généralisées à une connaissance, des attitudes et idéologies plus générales, privilégiées » (van Dijk 2013 : 250 , traduit par nous). Ce processus tire parti de l'inégalité de l'accès à l'information.

Il ne faut pas oublier la nature générale du pouvoir, qui implique l'aspect de la «capacité d'imposer et de maintenir une structuration particulière d'un domaine ou d'un autre - une manière particulière de le diviser en parties, de maintenir les parties délimitées les unes des autres, et un ordre particulier de ces parties en termes de relations hiérarchiques de domination et subordination » (Fairclough, 2003:13, traduit par nous). Cet aspect du pouvoir se manifeste dans les actions discursives de la presse sur le thème des réfugiés.

\subsubsection{L'hégémonie}

Selon Gramsci (1971, cité dans Flowerdew $2013: 183$, traduit par nous) l'hégémonie est « l'exercice du pouvoir par des moyens implicites plutôt que par la force militaire ». La sélection des sujets, les images choisies et leur impact visuel sur les histoires sont quelques exemples de l'influence de la presse sur l'opinion publique. Ces pratiques constituent un dispositif hégémonique souvent négligé, auquel on peut ajouter, comme mentionné précédemment, les techniques linguistiques comme la métaphore, utilisées pour couvrir les évènements : «Les changements dans la société se reflètent dans les changements de la pratique discursive et vice versa » (Flowerdew $2013: 183$, traduit par nous). Cette notion de combinaison de l'hégémonie et du discours est appelée hégémonie discursive, définie par Fairclough (2003, cité dans Flowerdew 2013: 183, traduit par nous) comme «la domination et la naturalisation de représentations particulières, la façon dont certains discours s'imposent dans des contextes sociopolitiques donnés, suite à une lutte entre les acteurs politiques pertinents ». Il y a également d'innombrables façons conscientes et inconscientes de contrôler un message. Il ne s'agit pas ici d'étudier l'origine de la crise des réfugiés, qui a pu en effet impliquer la force militaire, mais de se concentrer sur le langage utilisé dans la presse ainsi que sur le pouvoir hégémonique exercé par le biais de dispositifs linguistiques. Employés dans les reportages sur les réfugiés dans les pays hôtes, ces derniers risquent d'entraîner également des conséquences dommageables et de longue durée. 


\subsubsection{L'identité} les groupes, et qui dépend également de facteurs internes comme externes. Cette étude examine le concept d'identité du groupe qui est étiqueté réfugié et les facteurs externes qui façonnent cette identité, en particulier la presse. L'identité est «importante en termes de discours parce que l'identité se manifeste dans la pratique sociale, dont une partie importante est la pratique discursive " (Flowerdew 2013 : 184, traduit par nous). Bien qu'il ne s'agisse pas d'une étude psychologique, il est important de noter que l'identité sociale des réfugiés semble influencer leurs difficultés psychologiques, selon Kristal-Andersson (2000: 27), ce qui entraîne des difficultés d'adaptation supplémentaires au nouveau pays. Ces identités sociales sont formées à partir du pays d'origine et du pays d'accueil. L'identité est dans un état de flux constant et multiforme (Burgess et Ivanic 2010), construite par l'individu et par les autres, ainsi que par la manière dont il est perçu (Flowerdew $2013: 184$ ). Il est intéressant d'examiner la façon dont l'identité des réfugiés est façonnée dans et par la presse, par le biais d'un choix de collocations et de la prosodie sémantique qui en résulte.

Tous ces concepts de l'analyse critique du discours, d'idéologie, de pouvoir, d'hégémonie et d'identité doivent être pris en compte dans la méthodologie d'étude du discours des réfugiés par le biais des collocations. L'analyse critique du discours offre un excellent moyen d'analyser les données. Cependant, elle n'est pas sans limites ; l'une des deux principales limites est celle de la collecte de données et du biais. Le choix des sources et des méthodes de collecte de données est un exemple d'un problème avec collecte de données. Il peut y avoir des biais non seulement dans la sélection des textes, mais aussi dans l'analyse du texte. Pour pallier ces problèmes, une approche plus quantitative est nécessaire. Par conséquent, l'examen de ces concepts par l'analyse critique du discours pourrait ne pas suffire. L'analyse critique du discours peut être assistée par la linguistique de corpus, afin de simplifier le processus de collecte et de gestion des données et ainsi d'aider à éliminer les biais.

\subsection{La linguistique de corpus}

Contrairement à l'analyse critique du discours qui, à un niveau différent, se concentre sur la linguistique qualitative, la linguistique de corpus est surtout utilisée pour se concentrer sur la linguistique quantitative. Elle peut cependant être à la fois qualitative et quantitative, offrant de nombreux avantages :

Elle permet d'examiner de manière exhaustive les propriétés syntaxiques et sémantiques d'éléments lexicaux clés; elle peut servir d'heuristique, en fournissant des idées pour de nouvelles recherches qualitatives; elle produit des "résultats", la fréquence de certaines formes ou de certains collocations peut en soi être pertinente pour un point de vue critique ; la concordance est un outil de recherche extrêmement utile pour l'analyse des données plus efficacement que cela ne serait le cas autrement. Hardt-Mautner (1996, cité dans Flowerdew 2013 : 188, traduit par nous)

La linguistique de corpus nous permet d'examiner de grandes quantités de données et ainsi de les analyser, ce qui n'était pas possible auparavant. De plus :

les corpus sont aussi la seule source possible de données sur les propriétés statistiques des divers éléments des langues et sous-langues : leur distribution, la 
fréquence des lettres, les phonèmes, les mots, les catégories, les structures, les cooccurrences, leurs séquences et relations. (Bindi 1994 : 29, traduit par nous)

41 l'étude du discours des réfugiés par le biais de la collocation, dont il sera question plus loin.

\subsection{Analyse du discours critique assistée par la linguistique de corpus}

Une branche croissante de la méthodologie de recherche en linguistique appliquée consiste à combiner l'utilisation des méthodes qualitative et quantitative, dans l'espoir d'offrir le meilleur des deux approches (Dörnyei $2007: 20$, traduit par nous). Grâce à la linguistique de corpus, l'analyse du discours est le résultat d'un changement de paradigme par rapport à la méthodologie traditionnelle de l'analyse textuelle. Les nouvelles technologies simplifient en effet l'examen des corpus en analyse de texte. C'est le résultat des travaux de chercheurs de l'Université de Lancaster, dont ceux de Baker et al. (2008, traduit par nous) dans «Refugees and Asylum Seekers in the UK Press 1996-2006». L'étude de Baker et McEnery a démontré comment les corpus peuvent « jouer un rôle important dans la recherche sociale critique en permettant aux chercheurs d'identifier objectivement des modèles généralisés de langage naturel et des exemples rares mais révélateurs de l'existence de discours qui pourraient autrement être invisibles » (Baker et McEnery 2005 : 198, traduit par nous).

Stubbs souligne l'importance de l'utilisation des corpus comme outils dans la procédure, sans négliger l'élément humain :

Les procédures quantitatives peuvent identifier des ensembles lexicaux largement sur la base de la fréquence et de la distribution des éléments lexicaux dans un corpus, laissant l'analyste humain rejeter quelques collocations non pertinentes que la procédure provoque (à cause du contexte idiosyncrasique des corpus) et interpréter les ensembles lexicaux obtenus. (Stubbs 1995 : 20, traduit par nous)

De nombreux chercheurs apprécient la valeur du corpus linguistique comme une approche pour aider les études discursives. Pour paraphraser Partington (2008 : 97), en combinant une approche quantitative de nature statistique avec une approche qualitative du discours, il peut être possible d'accéder à des significations non évidentes.

Schnörch (2014 : 358) souligne que la description lexicographique différenciée, fiable et exigeante sur le plan qualitatif de mots ou de phrases, exige des méthodes sur corpus pour pouvoir traiter le volume de données. Bien que «l'utilité de l'utilisation des approches la linguistique de corpus dans l'analyse critique du discours ait déjà été démontrée (Baker 2004, 2006 ; Hardt-Mautner 1995 ; Koller et Mautner 2004 ; Mautner 2000 ; O'Halloran et Coffin 2004), il faut aussi noter que dans la plupart de ces études, l'utilisation des méthodes et cadres théoriques traditionnels associés à l'analyse critique du discours et la linguistique de corpus ne s'équilibre pas » (Baker et al. 2008 : 275 , traduit par nous). Les chercheurs ont choisi les parties des méthodologies qui correspondaient à leurs objectifs :

Lorsque les collocations sont examinées dans le cadre de la recherche de l'analyse critique du discours, elles ne sont habituellement pas calculées statistiquement, mais établies manuellement au moyen de concordances triées et de renseignements concernant leur signification statistique, l'intervalle de collocation ou tout seuil de 
fréquence ne sont habituellement pas fournis. (Baker et al. $2008: 275$, traduit par nous)

\section{suivantes :}

Commencer par une concordance générée par machine avec un grand corpus; une liste est compilée par ordre de fréquence, de toutes les formes de mots dans la concordance ; traiter la liste en l'ajustant pour inclure un intervalle de un à cinq mots de chaque côté du nœud, déterminer un point de coupure (moins de dix pour cent de fréquence par exemple), donner un poids aux autres collocations en fonction de sa fréquence dans la concordance de leur fréquence globale dans le corpus complet, examiner chaque ligne en ajoutant une cote et recourir aux cas les plus courants au début. (Sinclair 1991 : 104-105, traduit par nous)

Bon nombre des étapes de cette procédure peuvent maintenant être exécutées à l'aide d'un logiciel. Outre les approches de la linguistique de corpus et de l'analyse critique du discours, il existe un outil important pour calculer et fournir des données statistiques. Sketch Engine utilise des algorithmes pour aider les linguistes, chercheurs, traducteurs et autres à analyser de grandes quantités de données. Blinder et Allen, dans leur étude sur les immigrants illégaux (Blinder et Allen 2016 : 15), ont utilisé Sketch Engine et son système de classement, GDEX, pour illustrer les principaux résultats quantitatifs.

Plusieurs outils sont utilisés pour recueillir, trier et analyser les données. Dans cette étude, Sketch-Engine est utilisé dans ce travail de collecte et de tri. Cet outil de corpus 
prend en entrée un corpus de n'importe quelle langue et de motifs grammaticaux correspondants, et génère des croquis pour les mots de cette langue (Kilgarriff, Rychlý, Smrž, Tugwell 2004: 105). Au cours du processus de collecte et de tri des données, celles-ci sont examinées par concordances. Les collocations ou les c-collocations ${ }^{3}$ pour cette étude sont localisées, suivant la politique de Gabrielatos et Baker (Gabrielatos/ Baker 2008 : 11). L'analyse des données ne peut se faire que partiellement par logiciel. Celui-ci a été utilisé pour générer des concordances et, un intervalle de cinq mots à droite et à gauche du nœud a été analysé afin de fournir des collocations possibles, bien que Sketch Engine utilise à la fois l'algorithme t-score et le score d'information mutuelle (MI). L'algorithme du score $t$ mesure le niveau de confiance de l'association revendiquée. La fréquence élevée des mots grammaticaux (Clear 1993 : 281) constitue l'une des limites associées à cette situation. Sketch Engine utilise un autre outil, le score MI, qui montre dans quelle mesure la fréquence de cooccurrences observées diffère de ce que l'on pourrait prédire, également limité du fait que les collocations atypiques et particulières sont identifiées et que le meilleur résultat est une liste de collocations potentielles (Clear 1993: 282). Dans chaque cas ambigu, l'extrait produit par la concordance a été élargi afin de fournir des preuves textuelles supplémentaires, contexte à l'appui, de toute interprétation faite.

Une critique majeure de l'utilisation d'outils de concordance, comme Sketch Engine, utilisé dans cette étude, tient à ce qu'ils ne peuvent être utilisés que pour des données quantitatives. Dans le tableau suivant, Mautner (2015: 162) résume l'utilisation des concordanciers en présentant les deux types de preuves, quantitatives et qualitatives, qu'elles fournissent.

\begin{tabular}{ll}
\hline Quantitative evidence & Frequency lists \\
& Comparisons of wordlists, giving information on relative frequency \\
& ('keyness') \\
& Measures of statistical significance: \\
- t-score & - 'Mutual Information' (MI) score \\
Qualitative evidence & Concordance lines sorted alphabetically, enabling the researcher \\
& to identify \\
& - semantic preference \\
\hline
\end{tabular}

Tableau 1. Preuves quantitatives et qualitatives fournies par les concordanciers (Mautner 2015 : 162)

Un aspect clé de l'étude est la source des données. Quatre corpus ont été utilisés pour cette étude et ont été compilés à partir d'un algorithme qui parcourait continuellement 350000 flux RSS et utilisait Google News comme source d'articles, parfois en élaguant manuellement la liste; les articles ont été téléchargés à raison d'un par minute (Trampus : 2012, traduit par nous). Les données ont été recueillies entre 2014 à 2017. Il est important de noter la taille des corpus utilisés. Avec 36580048048500 tokens, le corpus anglais était le plus important. Viennent ensuite le corpus espagnol avec 6896969220 tokens, le corpus allemand avec 3462593757 tokens et le corpus français avec 3228931433 tokens ${ }^{4}$. L'évolution et la disponibilité même de ces corpus multiples peuvent non seulement nous donner une image plus complète et plus précise du langage et du discours utilisé, mais également transformer le sens du langage et du discours en termes et en concepts, dans la linguistique théorique et appliquée (de Beaugrande $2001: 3$ ). 

cette étude, elle a révélé à la fois des similitudes et des différences dans les corpus. Les similitudes dans les corpus résidaient dans l'utilisation d'un même champ sémantique. Dans les quatre corpus, les mots les plus fréquents partageant le même champ sémantique que le mot réfugié était une forme ou une autre du mot migrant. En anglais, en allemand et en français, c'était exactement le mot désignant 'migrant' ; le formulaire en espagnol comprenait les préfixes in- et $e$ - pour former inmigrante ('immigrant') et emigrante ('émigrant'). Une autre similitude entre l'anglais, l'allemand et le français est le mot demandeur d'asile, qui apparaît très fréquemment dans tous les corpus, sauf en espagnol. Les techniques et les outils de la linguistique de corpus sont les mieux adaptés pour obtenir de tels résultats, comme le montre le tableau suivant.

\begin{tabular}{|l|l|l|l|}
\hline Anglais & Allemand & Français & Espagnol \\
\cline { 1 - 2 } & $\begin{array}{l}\text { Asylbewerber } \\
\text { ('demandeur d'asile') }\end{array}$ & migrant & inmigrante (immigré) \\
\hline asylum seeker (asile) & Migrant & asile & emigrante (émigrant) \\
\hline immigrant (immigré) & & & \\
& & &
\end{tabular}

Tableau 2. Résultats - Champ sémantique

Ce tableau donne un exemple du type d'information que nous pouvons obtenir grâce à la linguistique de corpus. Nous pouvons l'utiliser pour recueillir, trier et gérer des informations statistiques. La linguistique de corpus peut nous aider à localiser des mots qui sont utilisés de manière similaire, situés dans le même champ sémantique. Elle peut être utilisée pour comparer ces données entre deux langues. Il en ressort que bien que le mot solic itante de asilo ('demandeur d'asile') existe en espagnol et se trouve dans le même champ sémantique que refugiado ('réfugié'), les exemples dans le corpus espagnol ne se retrouvent pas aussi souvent que dans les corpus anglais, allemand et français. À 
partir de ces informations, nous pouvons tirer quelques conclusions sur l'utilisation du mot dans la presse espagnole par rapport à la presse d'autres pays.

Une fois que la linguistique de corpus nous a permis de localiser les mots pouvant être utilisés à la place de celui de réfugié, elle peut également nous aider à trouver facilement des collocations. Pour déterminer les collocations les plus pertinentes sur le plan statistique pour le mot réfugié, les outils de la linguistique de corpus ont été utilisés. Dans une liste de collocations potentielles pour le mot réfugié, produite par le système, les quatre corpus, représentant les quatre langues, présentaient quelques similitudes. L'étude étant toujours en cours, seuls quelques exemples sont fournis ici. Par exemple, le modificateur syrien pour décrire les réfugiés était le plus courant dans ces quatre corpus. Les corpus anglais, français et espagnol contenaient tous une forme ou une autre du mot migrant dans une liste de collocations. Ainsi, après avoir examiné quelques exemples, nous pouvons voir que le mot migrant est souvent utilisé à la fois comme synonyme et comme modificateur du mot réfugié dans les corpus anglais, français et espagnol.

Les différences constatées dans chacun des corpus pouvaient être interprétées comme des façons différentes de communiquer des informations sur les réfugiés dans ces langues. Elles peuvent également être représentatives des caractéristiques distinctes de ces langues. Par exemple, le corpus anglais ne se contente pas de décrire la situation comme une crise, il évoque l'emplacement des réfugiés dans les camps, ce qui est plus rare dans les corpus des autres langues. Le corpus allemand mentionne moins la situation ou l'emplacement qu'il n'apporte d'informations démographiques sur les réfugiés eux-mêmes ou sur l'âge des réfugiés jung ('jeune'), minderjährig ('mineur'), par exemple. Il s'intéresse également aux mineurs, en particulier à ceux non accompagnés.

61 Le nom acogida revient fréquemment dans le corpus espagnol. Il est apparu comme une collocation fréquente et a de nombreuses utilisations et traductions en français. Dans le contexte du discours sur les réfugiés, il est souvent traduit en français par accueil, comme dans « l'accueil des réfugiés », y compris l'accueil et l'acceptation des réfugiés. L'utilisation supplémentaire se situe dans le même champ sémantique que le mot refugiado ('réfugié') lui-même, ce qui justifie sa fréquence d'apparition dans le corpus. Toutefois, l'examen des thèmes des corpus dans le contexte de l'analyse critique du discours peut fournir encore plus d'informations.

Pouvoir facilement trouver, trier et visualiser les collocations les plus fréquents pour le mot réfugié n'est qu'un début. Comprendre ce que cela signifie par rapport aux questions de recherche constitue l'étape suivante Cet article présente par la suite une analyse préliminaire des collocations. De plus, l'analyse critique du discours est utilisée pour identifier les thèmes de chaque corpus.

63 Certains thèmes se sont révélés récurrents dans chacun des corpus. Cependant, la fréquence à laquelle ils apparaissaient variait et, à première vue, chaque corpus semblait contenir des thèmes très différents. Il est toutefois intéressant de noter que tous les corpus avaient plusieurs thèmes en commun, la quantification, le mouvement, le crime et la nuisance pour n'en nommer que quelques-uns, mais à des fréquences variables. Par exemple, une certaine forme de quantification est apparue dans tous les corpus. On note ainsi l'utilisation de quantificateurs comme: plus, des millions, des milliers et la plupart. En fait, la quantification était le thème le plus fréquent dans le corpus anglais. Le titre d'un article du Guardian «Syrian refugees : more than $5 \mathrm{~m}$ in neighbouring countries now, says UN » (Weaver 2017, traduit par nous) en est un bon 
exemple. Le deuxième thème le plus fréquent dans trois des quatre corpus était le mouvement. Dans le corpus allemand, le mouvement était le quatrième thème le plus souvent abordé. Dans le corpus français, la criminalité et les nuisances étaient un thème important, comme en témoignent des collocations comme le statut dans les discussions portant non seulement sur les mises à jour, mais également sur la situation juridique des réfugiés, ainsi que sur la question de leur entrée légale ou non dans le pays. De nombreuses recherches linguistiques se sont concentrées sur ces thèmes et sur les métaphores qui s'y rapportent. Néanmoins, d'autres thèmes ont été identifiés, de sorte que tous les corpus contenaient des thèmes liés à l'aide ou à l'assistance aux réfugiés. En réalité, ces thèmes étaient plus importants dans le corpus espagnol, constitué de nombreux articles sur l'hébergement, l'accueil ou l'acceptation des réfugiés.

En ce qui concerne la prosodie sémantique, de nombreuses connotations et associations non neutres ont été relevées dans les collocations de réfugiés. Par exemple, le concept même de quantification a une connotation négative implicite d'objectivation lorsqu'il est utilisé pour décrire les gens. De plus, lorsque les quantificateurs pointent vers de grands nombres, ils sont souvent conçus pour choquer le lecteur, l'accabler de chiffres. Ce n'est qu'une interprétation parmi d'autres, mais l'effet pèse lourdement sur le nœud réfugié, produisant une prosodie sémantique négative globale. De plus, l'utilisation de mots concernant le statut des réfugiés comme anerkannt ('reconnu') dans le corpus allemand, qui ont souvent été collocalisés et modifiés avec le nœud réfugié, provoque le pléonasme sémantique réfugié reconnu. En effet, un réfugié a déjà demandé et obtenu le statut légal approprié pour rester dans le pays. Cependant, l'effet littéraire du pléonasme sémantique dans ce cas peut attirer l'attention et évoquer des images de personnes illégales entrant dans le pays, ce qui a un effet négatif. La prosodie négative peut aussi être conçue par l'utilisation de la métaphore. Décrire le mouvement comme l'afflux de réfugiés ou utiliser des métaphores similaires à celles que l'on trouve dans les quatre corpus est devenu si courant que l'on remarque à peine ses effets sur le lecteur.

De même, un auteur écrit d'un point de vue particulier et peut influencer grandement ses lecteurs. Le texte peut promouvoir intentionnellement ou involontairement une idéologie particulière ou un concept tel que la xénophobie ou l'islamophobie. On peut citer le cas du voile comme exemple d'écriture islamophobe liée aux réfugiés. Ajouter le modificateur islamique, créant ainsi les mots voile islamique, crée une relation dichotomique automatique entre nous et eux, entre le voile normalement associé aux mariages chrétiens ou catholiques, par exemple, et le voile islamique, qui serait associé à l'Islam. Un article de la BBC intitulé « Le voile islamique à travers l'Europe » (2018) en est un exemple. Une situation similaire s'est produite lorsque la BBC, dans l'article «Entrée en vigueur de l'interdiction du voile intégral dans les lieux publics en Autriche ", a utilisé le modificateur musulman pour créer les mots voile musulman (BBC 2017, traduit par nous). L'intérêt particulier porté dans ces articles à l'interdiction de la burka et plus généralement à l'apparence vestimentaire des femmes musulmanes participe de cette association entre voile et oppression ; il n'est pourtant pas nécessaire de rappeler que les médias ne dénoncent pas la robe comme symbole d'oppression féminine, pas plus qu'ils ne suggèrent l'interdiction pour les hommes pratiquant le judaïsme de porter la kippah/yarmulke. 
66 aspects politiques, socioculturels et économiques généraux de la domination exercée par la presse dans cette situation. Cette domination s'exerce sur les individus dont parlent les articles, qui ont peu de contrôle sur ce qui est rapporté à leur sujet. L'exercice hégémonique de ce pouvoir crée souvent de nouveaux problèmes pour les réfugiés dans les pays d'accueil, ce qui porte atteinte à leur identité et, dans certains cas, à la sécurité qu'ils recherchent désespérément dans le pays hôte.

\section{Conclusion}

67

Dans l'étude du discours sur les réfugiés par le biais de collocations, une approche par méthodes intégrées peut s'avérer très efficace. Une approche intégrée peut inclure les méthodes quantitative et qualitative. Les outils et techniques d'analyse linguistique d'un corpus de textes peuvent être utilisés pour trier, gérer et aider l'analyse préliminaire des données. L'analyse critique du discours, d'autre part, est le processus qui consiste à porter un regard critique sur les données pour aider les chercheurs à analyser des données qualitatives et quantitatives. Par conséquent, une méthodologie qui inclut les méthodes, les outils et les techniques de chaque approche offre de nombreux avantages dans l'étude du discours des réfugiés.

\section{BIBLIOGRAPHIE}

AL FAJRI, Muchamad Sholakhuddin (2017) : "Hegemonic and Minority Discourses around Immigrants: A Corpus-Based Critical Discourse Analysis". Indonesian Journal of Applied Linguistics $7.2: 381-390$.

ASHLEY, Kirk. "The maps and charts that explain how Europe's refugee crisis developed in 2016". The Telegraph. (consulté le 10.12.2018).

BAKER, Paul, \& TONy MCENERY (2005) : “A corpus-based approach to discourses of refugees and asylum seekers in UN and newspaper texts". Journal of Language and Politics 4.2 : 197-226.

BAKER, John Paul (2015) : "Does Britian need any more doctors? Inter-analyst consistency and corpus-assited (critical) discourse analysis". Corpora, Grammar and Discourse : In Honour of Susan Hunston, Vol. 73 ed. by Nicholas Groom, Maggie Charles \& John Suganthi. Amsterdam, Philadelphia : John Benjamins, 283-300.

BAKER, Paul, Costas GABRIELATOS, Majid KHOSRAVInIK, Michał KRZYŻANOWSKI (2008) : “A useful methodological synergy? Combining critical discourse analysis and corpus lingusitics to examine discourese of refugees and asylum seekers in the UK press". Discourse \& Society 19.3 : 273-306.

BBC. (2017) : Austrian ban on full-face veil in public places comes into force. https://www.bbc.com/ news/world-europe-41457427 (consulté le 19.11.2018)

BBC. (2018) : The Islamic veil across Europe. https://www.bbc.com/news/world-europe-13038095 (consulté le 19.11.2018).

Linx, 13 | 2020 
BERRY, Mike, Inaki GARCíA-BLANCO \& Kerry MOORE (2016) : Press coverage of the refugee and migrant crisis in the $E U:$ A content analysis of five European countries. https://www.unhcr.org/56bb369c9.pdf (consulté le 10.12.2018).

BINDI, Remo, Nicoletta CALZOLARI, Monica MONCHINI, Vito PIRRELLI \& Antonio ZAMPOLLI (1994) : “ Corpora and computational lexica : integration of different methodologies of lexical knowledge acquisition". Literary and linguistic computing, 9-46 https://doi.org/10.1093/llc/9.1.29 (consulté le 26.07.2016).

BLINDER, Scott \& William L. ALLEN (2016) : “Constructing immigrants : Portrayals of migrant groups in British national newspapers, 2010-2012". International Migration Review 50.1 : 3-40.

BÖKE, Karin, Matthias JUNG, Thomas NIEHR \& Marin WENGELER (2000) : "Vergleichende Diskurslinguistik. Überlegungen zur Analyse internationaler und intralingualer Textkorpora". Einwanderungsdiskurse. Wiesbaden : Springer VS Verlag für Sozialwissenschaften, 11-36.

BOURGEAIS, Vincent (2016) : “Demandes d'asile dans les États membres de l'UE : Nombre record de plus de 1,2 million primo-demandeurs d'asile enregistrés en 2015”. Eurostat communiqué de press. https://ec.europa.eu/eurostat/documents/2995521/7203842/3-04032016-AP-FR.pdf/078f4e14-8bb7-45d2bdbf-8bb3881270b2 (consulté le 11.06.2018).

BURGESS, Amy \& Roz IVANIC (2010) : “Writing and being written : Issues of identity across timescales". Written communication 27(2) : 228-255.

CLEAR, Jeremy (1993) : "From Firth principles : computational tools for the study of collocation". Text and technology: In honour of John Sinclair ed. by Mona BAER, Gill FRANCIS, \& Elena TOGNINI-BONELLI. Amsterdam, Philadelphia : John Benjamins Publishing Company, 271-292.

COCO, Federica. (2018) : "Number of asylum applications to EU nearly halves in 2017" Financial Times. https://www.ft.com/content/1db1a8ec-2c26-11e8-9b4b-bc4b9f08f381 (consulté le 18.12.2018).

DE BEAUGRANDE, Robert (2001) : "Large Corpora, Small Corpora, and the Learning of "Language"". Small corpus studies and ELT : theory and practice Vol. 5 ed. by Mohsen GHADESSEY, AleX HENRY \& Robert. L. ROSEBERRY. Amsterdam, Philadelphia : John Benjamins Publishing, 3-28.

DIJK, Teun van (1993) : "Principles of Critical Discourse Analysis". Discourse and Society, 4(2) : 249-283

DIJK, Teun van (1996) : "Discourse, power and access". Texts and practices : Readings in critical discourse analysis, ed. y Carmen Rosa Caldas-Coulthard \& Malcolm Coulthard : London and New York : Routledge, 84-104.

DÖRNYEI, Zolán (2007) : Research methods in applied linguistics. Berkeley : Oxford University Press. EUROSTAT (2018) : https://ec.europa.eu/eurostat/documents/2995521/8754388/3-20032018-APEN.pdf/50c2b5a5-3e6a-4732-82d0-1caf244549e3 (consulté le 12.12.2018).

FAIRCLOUGH, Norman (2003) : Analysing discourse : Textual analysis for social research. London: Routledge.

FERNÁNDEZ, Cayetano y Alfonso CORRAL (2016) : “La representación mediática del inmigrante magrebí en España durante la crisis económica (2010-2011)”. Migraciones internacionales 8.4 : 73-103.

FIRTH, John Rupert (1968) : “A Synopsis of Linguistic Theory, 1930-55”. Selected Papers of J.R. Firth (1952-59) ed. by Frank Robert PALMER. London : Longmans, 168-205. 
FIRTH, John Rupert (1957) : Papers in Linguistics 1934-1951. Oxford : Oxford University Press. FLOWERDEW, John (2013) : Discourse in English language education. London : Routledge. FOTOPOULOS, Stergios. \& Margarita KAIMAKLIOTI (2016) : "Media discourse on the refugee crisis : on what have the Greek, German and British press focused?". European View 15.2 : 265-279.

GABRIELATOS, Costas \& Paul BAKER (2008) : "Fleeing, sneaking, flooding : A corpus analysis of discursive constructions of refugees and asylum seekers in the UK press, 1996-2005". Journal of English linguistics 36.1 : 5-38.

GREENBAUM, Sidney (1974) : "Some Verb-Intensifier Collocations in American and British English". American Speech, vol. 49, no.1/2: 79-89.

HALLIDAY, Michael A.K. (1966) : "Lexis as a linguistic level". In Memory of JR Firth ed. by Charles E. BAZELL, \& John Rupert FIRTH. London : Longmans, 148-162.

HARDT-MAUTNER, Gerlinda (1995) : “'Only Connect.' Critical Discourse Analysis and Corpus Linguistics". Unit for Computer Research on the English Language Technical Papers 6, Lancaster University, http://ucrel.lancs.ac.uk/papers/techpaper/vol6.pdf (02.01.2018).

HOEY, Michael (1991) : Patterns of lexis in text (Vol. 299). Oxford : Oxford Press.

HOYER, Ariana (2016) : “Spanish News Framing of the Syrian Refugee Crisis”. WWU Honors Program Senior Projects 26. https://cedar.wwu.edu/wwu_honors/26

HUNSTON, Susan (2002) : Corpora in applied linguistics. Stuttgart : Ernst Klett Verlag.

IBRAHIM, Monica (2017) : “'Rapefugees Not Welcome' Ideological articulations of media discourses on migrants and refugees in Europe : New racisim and other - A critical discourse analysis" http://www.lse.ac.uk/media-and-communications/assets/documents/research/mscdissertations/2016/Dissertation-Monica-Ibrahim.pdf (consulté 10.12.2018).

JACQUEZ, Lisa (2015) : “De la difficulté de défendre les sans-papiers dans l'espace public français : typologie et analyse des contrediscours militants (2006-2010) ". Semen. Revue de sémiolinguistique des textes et discours 39. https://doi.org/10.4000/semen.10482

KHOSRAVINIK, Majid (2009) : “The representation of refugees, asylum seekers and immigrants in British newspapers during the Balkan conflict (1999) and the British general election (2005)". Discourse \& Society 20.4 : 477-498.

KILGARRIFF, A. (2005) : “Language is Never, Ever, Ever, Random”. Corpus Linguistics and Linguistic Theory 1(2) : 263-276.

KILGARRIFF, Adam, Pavel RYCHLÝ, Pavel SMRŽ, \& David TUGWELL (2004) : "Iltri-04-08 the sketch engine ". Information Technology 116 : 105-116.

KRISHNAMURTHY, Ramesh (2000) : “Collocation : from silly ass to lexical sets". Words in Context : A Tribute to John Sinclair on his Retirement ed. Chris HEFFER \& Helen SAUSTON. Birmingham : University of Birmingham, 31-47.

KRISTAL-ANDERSSON, Binnie (2000) : Psychology of the refugee, the Immigrant and their Children :

Development of a Conceptual Framework and Application to Psychotherapeutic and Related Spport Work. Drakenbergsgatan 63, 11741 Sotckholm : Office of Psychotherapy and Psychology in Stockholm South. 
Louw, Bill (2000) : "Contextual prosodic theory : Bringing semantic prosodies to life". Words in context : A tribute to John Sinclair on his retirement ed. by Chris HEFFER \& Helen SAUNSTON.

Birmingham : University of Birmingham, 48-94.

MAUTNER, Gerlinda (2015) : "Checks and balances : How corpus linguistics can contribute to CDA". Methods of critical discourse studies ed. by Ruth WODAK \& Michael MEYER. Thousand Oaks, CA : Sage Publications, 154-179.

MCENERY, Anthony M., \& Anita WILSON (2001) : Corpus linguistics : an introduction. Edinburgh : Edinburgh University Press.

MCENERY, Tony, Richard XIAO \& Yukio TONO (2006) : Corpus-based language studies : An advanced resource book. Abingdon : Taylor \& Francis.

MOORE, Kerry (2013) : “'Asylum shopping' in the neoliberal social imaginary”. Media, Culture \& Society $35.3: 348-395$.

MUSTAFA-AWAD, Zahra \& Monika KIRNER-LUDWIG (2017) : “Arab women in news headlines during the Arab Spring : Image and perception in Germany". Discourse \& Communication 11.5 : 515-538.

PARKER, Samual. (2015) : “'Unwanted invaders' : The representation of refugees and asylum seekers in the UK and Australian print media". eSharp 23.1 : 1-21.

PARTINGTON, Alan (1998) : Patterns and meanings : Using corpora for English language research and teaching Vol. 2. Amsterdam, Philadelphia : John Benjamins Publishing.

PARTINGTON, Alan (2008) : "The armchair and the machine : Corpus-assisted discouse research". Corpora for university language teachers, ed. by C. Taylor Torsello". Bern et al. : Peter Lang, 95-118.

POUSHTER, Jacob. (2018) Pew Research Center : "European opinions of the refugee crisis in 5 charts" http://www.pewresearch.org/fact-tank/2016/09/16/european-opinions-of-the-refugeecrisis-in-5-charts (consulté le 10.12.2018).

SALAS, Luis Guerra (2016) : "La representacón de los movimientos migratorios en la prensa de los países hispanohablantes (2013-2015)”. Cuadernos AISPI. Estudios de lenguas y literaturas hispánicas 8 : 95-117.

SARDINHA, Tony Berber (2000) : "Semantic prosodies in English and Portuguese : A contrastive study". Cuademos de Filología Inglesa 9.1 : 93-109.

SCHNÖRCH, Ulrich (2014) : Uberlegungen zur (adäquaten) lexikografischen Beschreibung des ('politisch korrekten') Gebrauchs von Wörtern wie Ausländer, Migrant, Flüchtling, Asylbewerber. Institut für Deutsche Sprache (IDS). https://ids-pub.bsz-bw.de/frontdoor/deliver/index/docId/ 4133/file/ Schnoerch_Ueberlegungen_zur_(adaequaten)_lexikogra\%ef\%ac\%81schen_Beschreibung_des_(politisch_korrekten)_Gebrauchs_von_W (consulté le 10.12.2018).

SINCLAIR, John (1991) : Corpus, concordance, collocation. Oxford : Oxford University Press.

SINCLAIR, John, Susan JONES \& Rober DALEY (1970) : English Collocation Studies : The OSTI Report. London : Continuum.

STUBBS, Michael (1995) : "Collocations and semantic profiles : On the cause of the trouble with quantitative studies". Functions of language 2.1 : 23-55.

TOGNINI-BONELLI, Elena (2001) : Corpus linguistics at work Vol. 6. Amsterdam, Philadelphia : John Benjamins Publishing. 
TRAMPUS, Mitja \& Blaž NOVAK (2012) : “Internals of an aggregated web news feed". Proceedings of $15^{\text {th }}$ Multiconference on Information Society (IS-2012).

VAN DIJK, Teun (2013) : “Discourse, power and access”. Texts and practices, Routledge, 93-113.

WEAVER, Matthew (2017) : "Syrian refugees : more than $5 \mathrm{~m}$ in neighbouring countries now, says

UN”. The Guardian. Extrait le 14 novembre 2018 : https://www.theguardian.com/world/2017/

mar/30/syrian-refugee-number-passes-5m-mark-un-reveals.

WODAK, Ruth (2009) : Methods for critical discourse analysis. Thousand Oaks, CA : Sage

XIAO, Richard \& TONy MCENERY (2006) : “Collocation, semantic prosody, and near synonymy : A

cross-linguistic perspective”. Applied linguistics $27.1: 103-129$.

\section{NOTES}

1. Son analyse s'appuie sur les sources des bases de données Nexis et Newsbank.

2. Il fait référence aux pratiques journalistiques. Pour plus de détails sur l'alimentation et l'accès à l'alimentation, voir van Dijk (2013).

3. Les c-collocations sont des collocations statistiquement «consistent" dans le temps et non saisonnières, par exemple.

4. Les données du site web Sketch Engine. https://www.sketchengine.eu/ (01.04.2018)

\section{RÉSUMÉS}

Cet article examine l'efficacité de l'intégration de méthodes et d'approches quantitatives et qualitatives pour étudier le discours des réfugiés par les collocations (cooccurrences). Les approches statistiques (quantitatives) et les outils et techniques de linguistique de corpus ont aidé à l'analyse préliminaire et fournit une heuristique pour la découverte de modèles sur des données provenant de la presse anglaise, française, allemande et espagnole. L'analyse critique du discours a été utilisée pour analyser les mots fréquents, les collocations, les thèmes (sujets) et la prosodie sémantique. Des modificateurs liés à l'âge des réfugiés, Jung (jeune), minderjährig (mineur), ont été trouvés dans un corpus (allemand) tandis que des modificateurs juridiques, statut, politique, ont été trouvés dans un autre corpus (français). Les quatre corpus comprenaient les collocations de réfugiés syriens attendues compte tenu des phénomènes. Étaient récurrents les quantificateurs de plus, des millions et des milliers et des métaphores de mouvement combinées à des quantificateurs (anglais) enormous influx ('énorme afflux') ou large influx ('grand afflux') et (espagnol) la gran afluencia ('un grand afflux'). Les thèmes du crime et de la nuisance, comme en témoignent les verbes fréquents (français) emprisonner, arrêter et déporter ont démontré la prosodie sémantique négative saillante.

This article discusses the effectiveness of integrating quantitative and qualitative methods and approaches to study refugee discourse through collocations (co-occurrences). Statistical (quantitative) approaches and corpus linguistics tools and techniques assisted the preliminary analysis as well as provided a heuristic for pattern discovery on data from the English, French, German and Spanish press. Critical discourse analysis was used to analyze frequent words, 
collocations, themes (topics) and semantic prosody. Modifiers related to the age of refugees were found in one corpus (German) Jung (young), minderjährig (underaged), whereas legal modifiers were found in another (French s) statut (status), politique (political). All four corpora included the collocations Syrian refugees, expected considering the phenomena. Quantifiers were recurrent more, millions, and thousands, movement metaphors combined with quantifiers (English) enormous influx or large influx and (Spanish) la gran afluencia (a large influx). Crime and nuisance themes as evidenced in frequent verbs (French) emprisonner (imprison), arrêter (arrest), and déporter (deport) demonstrated the salient negative semantic prosody.

\section{INDEX}

Keywords : Text analysis, collocation, refugees, critical discourse analysis, corpus linguistics Mots-clés : Analyse de texte, collocation, réfugiés, analyse critique du discours, linguistique de corpus

\section{AUTEUR}

\section{JOY WEIGAND}

Université de Potsdam 